\title{
Nonsingular universe from generalized thermostatistics
}

\author{
B. Vakili ${ }^{1, *}$ and M. A. Gorji ${ }^{2, \dagger}$ \\ ${ }^{1}$ Department of Physics, Central Tehran Branch, Islamic Azad University, Tehran, Iran \\ ${ }^{2}$ Department of Physics, University of Mazandaran, P.O. Box 47416-95447, Babolsar, Iran
}

\begin{abstract}
We study the statistical mechanics of the early radiation dominated universe in the context of a generalized uncertainty principle which supports the existence of a minimal length scale. Utilizing the resultant modified thermodynamical quantities, we obtain a deformed Friedmann equation which is very similar to that arises from loop quantum cosmology scenarios. The energy and entropy densities get maximum bounds about Planck temperature and a nonsingular universe then emerges in this setup.
\end{abstract}

PACS numbers $04.60 . \mathrm{Bc}, 98.80 . \mathrm{Cq}$

Key Words Quantum Gravity Phenomenology, Thermodynamics, Cosmology

\section{Introduction}

Although the standard Big Bang model of cosmology describes our observable universe with high accuracy, its shortages are revealed when, for instance, the model is implemented to describe the early state of the universe. The initial condition (fine-tuning) problem emerges in the ultraviolet regime which can be resolved by considering an accelerated inflationary phase about $10^{15} \mathrm{GeV}$ [1]. Furthermore, the universe starts from an initial Big Bang singularity where the classical equation of general relativity fails to be applicable. The Big Bang singularity, however, appears at very high energy regime where the quantum effects will become significant and it is then natural to expect that the problem will be resolved in the framework of a quantum theory of gravity. Unlike electromagnetic, weak and strong nuclear interactions which are quantized very successfully by means of the gauge field quantization method, quantization of gravity is not an easy task. While the three other forces are classically described by the gauge fields that propagate on the fixed Minkowski background geometry, gravity itself is a background geometry and quantization of gravity is then equivalent to the quantization of geometry. Although a full quantum theory of gravity is not formulated yet, the elegant work of Ashtekar in Ref. [2] inspired the loop representation of the quantum general relativity [3] (for review see Ref. 4]). Very interestingly, the Big Bang singularities are replaced by quantum bounces when loop quantum gravity method is applied into the homogeneous and isotropic spacetime in loop quantum cosmology scenario [5] (see Ref. [6] for review). Indeed, the volume operator is quantized with respect to a fundamental length scale (preferably of the order of Planck length) in loop quantum gravity [7] and, then, an ultraviolet cutoff appears which removes the Big Bang singularity. In fact, existence of a minimal length scale is a common address of any quantum theory of gravity such as string theory and loop quantum gravity 8. For instance, inspired by the string theory, the generalized uncertainty principle (GUP) is investigated which supports the existence of a minimal length through a nonzero uncertainty in position measurement [9, 10, 11. The statistical mechanics then will be significantly modified in the high energy regimes and the thermodynamics of the early universe will be affected by the GUP corrections [9]-[16. Since the GUP effects are related to the existence of a minimal length cutoff, it is natural to expect that this setup may resolve the Big Bang singularity [17, 18, 19. Motivated by this idea, we study the effects of GUP on the thermodynamics of the early universe and interestingly we find a modified Friedmann equation which is very similar to that arises from loop quantum cosmology.

The structure of the paper is as follows: In section 2, we formulate the generalized uncertainty principle and the idea of the existence of a minimal length from which we find the associated deformed density of states. In section 3 , we will study the thermodynamics of the early radiation dominated universe in GUP framework by means of the deformed density of states. In section 4, the modified Friedmann equations are obtained and the results are compared with those arise from loop quantum cosmology scenarios. Section 5 is devoted to the summary and conclusions.

\footnotetext{
*email: b.vakili@iauctb.ac.ir

$\dagger$ email: m.gorji@stu.umz.ac.ir
} 


\section{GUP and deformed density of states}

Since the standard Heisenberg uncertainty principle does not take into account any universal length scale, considering a minimal length scale will naturally lead to the modification of this principle. Nevertheless, there is not a unique deformation to the uncertainty principle. Here, we consider the generalized uncertainty relation as 11

$$
\Delta q \Delta p \geq \frac{1}{2}\left(1+(\beta \Delta p)^{2 n}\right),
$$

where $n \geq 1, \beta=\mathcal{O}(1) l_{\mathrm{P} 1}$, with $l_{\mathrm{P} 1}$ being the Planck length, is the deformation parameter and the dimensionless numerical coefficient $\mathcal{O}(1)$ should be fixed by the experiments [20]. In the $n=1$ case, relation (11) reduces to the well-known generalized uncertainty relation which is investigated in the context of the string theory [10]. Apart from some details of the various versions of such uncertainty relations, all of them should satisfy at least two properties: They should support the idea of existence of a minimal length scale and the corresponding uncertainty relations have to reduce to the standard Heisenberg uncertainty principle in the light of the correspondence principle. By a little algebra, it is easy to show that the proposed model (1) predicts a minimal uncertainty in position measurement as

$$
\Delta q_{\min }=2 n(2 n-1)^{\frac{1-2 n}{2 n}} \beta,
$$

from which it is seen that the uncertainty relation (11) results to a cutoff on minimal length. Also, it clearly reduces to the standard uncertainty principle in the low energy limit $\beta \rightarrow 0$.

By using of the standard (quantization scheme independent) definition $(\Delta A)^{2}(\Delta B)^{2} \geq \frac{1}{4}\langle[A, B]\rangle^{2}$, it is straightforward to show that the modified uncertainty relation (11) can be realized from the following deformed Heisenberg algebra

$$
[\hat{q}, \hat{p}]=\left(1+(\beta \hat{p})^{2 n}\right) .
$$

However, since in this work we are interested in the statistical mechanics of a many-particle system, we generalize this setup to the three dimensional case. A natural generalization which preserve the rotational symmetry will be

$$
\left[\hat{q}_{i}, \hat{p}_{j}\right]=\left(1+(\beta \hat{p})^{2 n}\right) \delta_{i j}
$$

where $\hat{p}^{2}=\hat{p}_{x}^{2}+\hat{p}_{y}^{2}+\hat{p}_{z}^{2}$. Because of the existence of a nonzero uncertainty in positions measurement, it is natural to suppose

$$
\left[\hat{p}_{i}, \hat{p}_{j}\right]=0
$$

which allows us to work in momentum representation with

$$
\hat{p}_{i} \cdot \psi(p)=p_{i} \psi(p), \quad \hat{q}_{i} \cdot \psi(p)=i\left(1+(\beta p)^{2 n}\right) \frac{\partial \psi(p)}{\partial p_{i}},
$$

where $p=\sqrt{p_{x}^{2}+p_{y}^{2}+p_{z}^{2}}$. The commutation relation between the position operators for the above representation then becomes

$$
\left[\hat{q}_{i}, \hat{q}_{j}\right]=2 i n \beta^{2 n} \hat{p}^{2 n-2}\left(\hat{p}_{i} \hat{q}_{j}-\hat{p}_{j} \hat{q}_{i}\right)
$$

where the results of the Ref. [10] can be correctly reproduced for the case $n=1$.

In order to obtain the density of states of a statistical system, we consider the classical limit of this setup. The deformed Heisenberg algebra which is defined by the commutation relations (4), (5) and (77) then can be extracted from the deformed non-canonical Poisson algebra

$$
\left\{q_{i}, q_{j}\right\}=2 n \beta^{2 n} p^{2 n-2}\left(p_{i} q_{j}-p_{j} q_{i}\right), \quad\left\{q_{i}, p_{j}\right\}=\left(1+(\beta p)^{2 n}\right) \delta_{i j}, \quad\left\{p_{i}, p_{j}\right\}=0,
$$

in which the operators are replaced by their corresponding phase space variables counterparts $\left(q_{i}\right.$ and $\left.p_{i}\right)$ and the Dirac commutators by the Poisson brackets. The invariant Liouville measure which is consistent with the non-canonical Poisson algebra (8) then will be [13, 14, 16]

$$
d^{3} q d^{3} p \rightarrow \frac{d^{3} q d^{3} p}{\left(1+(\beta p)^{2 n}\right)^{3}} .
$$

In Ref. [21], we have obtained the deformed Liouville measure for a generally deformed Poisson algebra and showed that it is invariant under the time evolution of the system which guaranties the validity of the Liouville theorem in such theories.

\footnotetext{
${ }^{1}$ We work in natural units $\hbar=c=k_{B}=1$, where $\hbar, c$ and $k_{B}$ are the Planck constant, speed of light in vacuum and Boltzman constant, respectively.
} 


\section{Thermodynamics of the early universe}

Having the deformed invariant Liouville measure (9) at hand, one can easily study the statistical mechanics in semiclassical regime in the context of the GUP (1).

By assuming a radiation dominated universe in early times of cosmic evolution, we consider the thermodynamics of the ultra-relativistic gas confined in volume $V$ at temperature $T$. The pressure is given by the standard definition

$$
\mathrm{P} V=\mp T \sum_{\varepsilon} \ln \left(1 \mp e^{-\varepsilon / T}\right),
$$

where the signs $(-)$ and $(+)$ refer to the bosons and fermions respectively and the summation is over the quantized energies of the microstates $\varepsilon$. At high temperature regime, the summation over $\varepsilon$ may be replaced by an integral over all phase space variables in the semiclassical regime by means of the invariant Liouville measure (9) as

$$
\sum_{\varepsilon} \rightarrow \frac{g V}{(2 \pi)^{3}} \int \frac{d^{3} p}{\left(1+(\beta p)^{2 n}\right)^{3}},
$$

where $g$ is the number of relativistic degrees of freedom and we have also performed the integral over the positions as $\int_{V} d^{3} q=V$. Now, the pressure in the GUP framework can be obtained from definition (10) and the deformed density of states (11) as

$$
\mathrm{P}_{\mp}=\mp \frac{g_{\mp} T}{2 \pi^{2}} \int_{0}^{\infty} \frac{\ln \left(1 \mp e^{-p / T}\right) p^{2} d p}{\left(1+(\beta p)^{2 n}\right)^{3}},
$$

where $g_{-}$and $g_{+}$are the number of relativistic degrees of freedom for bosons and fermions respectively and we have substituted $\varepsilon=p$ for the ultra-relativistic particles. Using the dimensionless variable $x=p / T$, the relation (12) becomes

$$
\mathrm{P}_{\mp}(T)=\frac{\pi^{2} T^{4}}{90} I_{\mp}(T),
$$

in which we have defined

$$
I_{\mp}(T)=\mp \frac{45}{\pi^{4}} g_{\mp} \int_{0}^{\infty} \frac{\ln \left(1 \mp e^{-x}\right) x^{2} d x}{\left(1+(\beta x T)^{2 n}\right)^{3}} .
$$

To evaluate the above integrals we may expand the denominator for $\beta p=\beta x T \ll 1$, for which by using of the integral formula

$$
\int_{0}^{\infty} \frac{x^{s-1} d x}{e^{x}+1}=\left(1-\frac{1}{2^{s-1}}\right) \int_{0}^{\infty} \frac{x^{s-1} d x}{e^{x}-1},
$$

the integrals in (14) can be evaluated as

$$
\begin{gathered}
I_{-}(T)=g_{-}\left[1-\frac{135}{\pi^{4}} \Gamma(2 n+3) \zeta(2 n+4) \beta^{2 n} T^{2 n}\right], \\
I_{+}(T)=\frac{7}{8} g_{+}\left[1-\frac{1080}{7 \pi^{4}} \Gamma(2 n+3) \zeta(2 n+4)\left(1-2^{-(2 n+3)}\right) \beta^{2 n} T^{2 n}\right] .
\end{gathered}
$$

These functions satisfy the conditions $I_{-}(T \rightarrow 0)=g_{-}$, and $I_{+}(T \rightarrow 0)=\frac{7}{8} g_{+}$. Thus, the usual results of the low temperature regime are recovered. Upon Substitution of (15) and (16) into relation (13), we get the total pressure $\mathrm{P}(T)=\mathrm{P}_{-}(T)+\mathrm{P}_{+}(T)$ as

$$
\mathrm{P}(T)=\mathrm{P}_{0}(T)\left[1-\chi(n) \frac{g_{*}(n)}{g_{0 *}} \beta_{0}^{2 n}\left(\frac{T}{T_{\mathrm{Pl}}}\right)^{2 n}\right],
$$

where $\mathrm{P}_{0}=\frac{\pi^{2} g_{0 *}}{90} T^{4}$ is the standard pressure for the ultra-relativistic gas, $g_{0 *}=g_{-}+\frac{7}{8} g_{+}$is the number of effective degrees of freedom, and we have also defined

$$
\begin{aligned}
& \chi(n)=\frac{135}{\pi^{4}} \Gamma(2 n+3) \zeta(2 n+4), \\
& g_{*}(n)=g_{-}+\left(1-2^{-(2 n+3)}\right) g_{+},
\end{aligned}
$$

with $\Gamma(y)$ and $\zeta(y)$ being the Gamma and Riemann Zeta functions respectively. 
The associated energy and entropy densities of the system can be obtained from their definitions

$$
\rho(T)=T^{2} \frac{\partial}{\partial T}\left(\frac{\mathrm{P}}{T}\right), \quad s=\frac{\rho+\mathrm{P}}{T},
$$

with results

$$
\begin{aligned}
& \rho(T)=\rho_{0}(T)\left[1-\left(1+\frac{2 n}{3}\right) \chi(n) \frac{g_{*}(n)}{g_{0 *}} \beta_{0}^{2 n}\left(\frac{T}{T_{\mathrm{Pl}}}\right)^{2 n}\right], \\
& s(T)=s_{0}(T)\left[1-\left(1+\frac{n}{2}\right) \chi(n) \frac{g_{*}(n)}{g_{0 *}} \beta_{0}^{2 n}\left(\frac{T}{T_{\mathrm{Pl}}}\right)^{2 n}\right],
\end{aligned}
$$

where $\rho_{0}(T)=3 \mathrm{P}_{0}=\frac{\pi^{2} g_{0 *}}{30} T^{4}$ and $s_{0}(T)=\frac{\rho_{0}+\mathrm{P}_{0}}{T}=\frac{2 \pi^{2} g_{0 *}}{45} T^{3}$ are the standard energy and entropy densities respectively. The relation (21) coincides with the result obtained in Ref. [15] when $n=1$. It is important to note that the GUP-thermodynamical quantities (17), (21) and (22) are not positive definite, but all of them are positive for $T<\left[\frac{\pi^{4}\left(g_{0 *} / g_{*}(n)\right)}{45 \Gamma(2 n+4) \zeta(2 n+4)}\right]^{\frac{1}{2 n}} \frac{T_{\mathrm{P} 1}}{\beta_{0}}$. In the following, we will see that the thermodynamical quantities approach to a maximum value below this temperature. We show that this corresponds to a nonsingular radiation dominated universe.

\section{Cosmological implications}

With the use of the deformed pressure (17) and energy density (21), we can easily consider the GUP effects on the evolution of the early universe. To do this, note that the energy-momentum tensor of the radiation $T_{\mu \nu}=\left(-\rho, \mathrm{P} \delta_{i j}\right)$ will be significantly modified at high temperature regime. For the spatially flat Friedmann-Robertson-Walker universe with metric $g_{\mu \nu}=\left(-1, a^{2}(t) \delta_{i j}\right)$, the Einstein's equations lead to

$$
H^{2}=\frac{8 \pi G}{3} \rho,
$$

where $H(t)=\frac{\dot{a}}{a}$ is the Hubble parameter, $a(t)$ is the scale factor and a dot denotes derivative with respect to the cosmic time $t$. This relation is nothing but the deformed Friedmann equation in GUP framework since the energy density is now modified through the relation (21). The conservation of the energy-momentum tensor $\nabla_{\mu} T_{\nu}^{\mu}=0$ also gives

$$
\dot{\rho}+3 H(\rho+\mathrm{P})=0,
$$

which is also deformed in GUP setup since the energy density and pressure are now given by the relations (21) and (17) respectively. Furthermore, bearing in the mind the well-known adiabatic condition

$$
S=s a^{3}=\text { constant, }
$$

the total entropy, $S$, of the universe is assumed to be constant in standard model of cosmology. Using the definitions (20), it is not difficult to show that the relations (24) and (25) are nothing other than the manifestation of the first law of thermodynamics. Substituting the GUP-modified energy density (21) into the relation (23) gives the deformed Friedmann equation in GUP framework as

$$
H^{2}=\frac{8 \pi G}{3} \rho_{0}\left[1-\left(\frac{\rho_{0}}{\rho_{c}(n)}\right)^{\frac{n}{2}}\right]
$$

in which we have defined the critical energy density

$$
\rho_{c}(n)=\left[\frac{\pi^{4}\left(g_{0 *} / g_{*}(n)\right)}{45 \Gamma(2 n+4) \zeta(2 n+4)}\right]^{\frac{2}{n}} \rho_{\mathrm{P} 1},
$$

with $\rho_{\mathrm{Pl}}=\frac{\pi^{2} g_{0 *}}{30} \frac{T_{\mathrm{P1}}^{4}}{\beta_{0}^{4}}$. For the case $n=1$, the GUP-modified Friedmann equation (26) will be reduced to the result that is obtained in Ref. [18] in which the authors studied the GUP effects in the framework of the Hamiltonian formalism of general relativity. However, it is important to note that while the conservation of energy remains unchanged in their setup, it will be modified in our formulation through the relation (24).

Now, we note that at the temperature $T_{*}=\left[\frac{2 \pi^{4}\left(g_{0 *} / g_{*}(n)\right)}{45(2+n) \Gamma(2 n+4) \zeta(2 n+4)}\right]^{\frac{1}{2 n}} \frac{T_{\mathrm{P} 1}}{\beta_{0}}$, the energy density (21) reaches to its maximum value $\frac{\rho_{c}}{2}$. The entropy density (22) also has maximum at this temperature and thus, according to the adiabatic condition (25) the scale factor will have a minimum as

$$
a_{\text {min }}=\left(S\left[\frac{45(3+2 n)}{4 n \pi^{2} g_{0 *}}\right]^{\frac{1}{3}}\left[\frac{45 g_{*}(n)}{2 \pi^{4} g_{0 *}}(2 n+1) \Gamma(2 n+4) \zeta(2 n+4)\right]^{\frac{1}{2 n}}\right) \beta_{0} l_{\mathrm{Pl}} .
$$


Therefore, in the case where GUP considerations are taken into account, we have a nonsingular cosmology in which the minimum of its scale factor occurs at the temperature $T_{*}$ for which $\rho_{0}=\left(1+\frac{n}{2}\right)^{-\frac{2}{n}} \rho_{c}(n)$. This means that the evolution of the scale factor based on the quantum gravity effects, shows a bouncing like behavior in such way that the classical big bang singularity will be replaced by a minimal size of the scale factor.

\subsection{The case $n=2$}

Let us now take a look at the special case $n=2$ for which the modified Friedmann equation

$$
H^{2}=\frac{8 \pi G}{3} \rho_{0}\left(1-\frac{\rho_{0}}{\rho_{c}(2)}\right)
$$

is very similar to that arises from loop quantum cosmology [5, 6. However, apart from this similarity, there is a crucial difference between them due to the holonomy corrections (see below). Here, the critical energy density $\rho_{c}(2)$ is defined from (27), that is

$$
\rho_{c}(2)=\left(\frac{g_{0 *}}{g_{*}(2)}\right) \frac{\rho_{\mathrm{P} 1}}{24 \pi^{4}},
$$

with $g_{*}(2)=g_{-}+\frac{127}{128} g_{+}$from (19). In this case, the GUP-deformed energy density (21) approaches to its maximum at $\rho_{0}=\frac{\rho_{c}(n)}{2}$ corresponds to the minimum value of the scale factor as

$$
a_{\min }=S\left(\frac{3 \pi \times 7 ! !}{4 g_{0 *}}\right)^{\frac{1}{3}}\left(5 ! ! \times \frac{g_{*}(2)}{g_{0 *}}\right)^{\frac{1}{4}} \beta_{0} l_{\mathrm{Pl}} .
$$

It is important to note that while the standard conservation relation $\dot{\rho}_{0}+3 H\left(\rho_{0}+\mathrm{P}_{0}\right)=0$ keeps its form in loop quantum cosmology, it is not the case in GUP formalism since here we have

$$
\dot{\rho}_{0}+3 H\left(\rho_{0}+\mathrm{P}_{0}\right)=2 \frac{\rho_{0}}{\rho_{c}}\left(\dot{\rho}_{0}+\frac{12}{7} H \rho_{0}\right),
$$

in which we have used the relations (17) and (21). This is because of the fact that, while the geometric part of the Einstein's equation gets holonomy corrections in loop quantum cosmology, by the GUP setting the matter part (energy-momentum tensor) will be modified. The relation (32) also shows that the matter may interact with itself in GUP framework.

\section{Summary}

Quantum gravity proposal suggests the existence of a minimal measurable length below which no other length can be observed. Taking a minimal length scale into account immediately leads to the modification of the standard uncertainty principle. In this paper, we first formulated a generalized uncertainty principle which supports the existence of a minimal length through the nonzero uncertainty in position measurement. For a statistical system, we obtained the associated deformed density of states and then implemented it in order to explore the minimal length effects on the thermodynamics of the radiation dominated universe. We showed that the GUP corrections to the pressure, energy density and entropy density of the universe yield to a maximum value for the energy and entropy densities at high temperature regime when the temperature becomes of the order of Planck temperature. By utilizing the modified thermodynamical quantities for the energy-momentum tensor, we obtained the modified Friedmann equation which seems to be similar to the Friedmann equation in loop quantum cosmology. We saw that the modifications due to the GUP considerations make a bouncing like behavior for the scale factor free of the classical big bang singularity. In addition to singularity avoidance, the appearance of a minimal size for the scale factor in the presented model is also interesting in its nature due to prediction of a minimal size for the corresponding universe. It is well-known that the idea of existence of a minimal length in nature is supported by almost all candidates of quantum gravity. Finally, we have noticed a difference between loop quantum cosmology and GUP results. While in the first theory the geometric part of the Einstein's equations gets holonomy corrections, the matter part is modified in the second. Thus, although the energy conservation relation remains unchanged in loop quantum cosmology, we showed it will be modified in GUP setup.

\section{References}

[1] A. H. Guth, Phys. Rev. D 23 (1981) 347

A. D. Linde, Phys. Lett. B 108 (1982) 389

A. Albrecht and P. J. Steinhardt, Phys. Rev. Lett. 48 (1982) 1220. 
[2] A. Ashtekar, Phys. Rev. Lett. 57 (1986) 2244

A. Ashtekar, Phys. Rev. D 36 (1987) 1587.

[3] C. Rovelli and L. Smolin, Nucl. Phys. B 331 (1990) 80

A. Ashtekar and J. Lewandowski, Class. Quantum Grav. 14 (1997) A55

A. Ashtekar and J. Lewandowski, Adv. Theor. Math. Phys. 1 (1998) 388

A. Ashtekar and J. Lewandowski, Class. Quantum Grav. 21 (2004) R53.

[4] C. Rovelli, Living Rev. Rel. 1 (1998) 1

C. Rovelli, Quantum Gravity, Cambridge University Press, Cambridge, UK, (2004)

T. Thiemann, Modern Canonical Quantum General Relativity, Cambridge University Press, Cambridge, UK, (2007).

[5] M. Bojowald, Phys. Rev. Lett. 86 (2001) 5227

M. Bojowald, Class. Quantum Grav. 19 (2002) 2717

A. Ashtekar, T. Pawlowski and P. Singh, Phys. Rev. Lett. 96 (2006) 141301

A. Ashtekar, T. Pawlowski and P. Singh, Phys. Rev. D 74 (2006) 084003

A. Ashtekar, T. Pawlowski and P. Singh, Phys. Rev. D 73 (2006) 124038.

[6] A. Ashtekar, M. Bojowald and J. Lewandowski, Adv. Theor. Math. Phys. 7 (2003) 233

M. Bojowald, Living Rev. Rel. 11 (2008) 4

A. Ashtekar and P. Singh, Class. Quantum Grav. 28 (2011) 213001.

[7] C. Rovelli and L. Smolin, Nucl. Phys. B 442 (1995) 593

R. Loll, Phys. Rev. Lett. 75 (1995) 3048

R. Loll, Nucl. Phys. B 460 (1996) 143

J. Brunnemann and T. Thiemann, Class. Quantum Grav. 23 (2006) 1289.

[8] D. J. Gross and P. F. Mende, Nucl. Phys. B 303 (1988) 407

D. Amati, M. Ciafaloni and G. Veneziano, Phys. Lett. B 216 (1989) 41

K. Konishi, G. Paffuti and P. Provero, Phys. Lett. B 234 (1990) 276

L. Garay, Int. J. Mod. Phys. A 10 (1995) 145.

[9] M. Maggiore, Phys. Lett. B 304 (1993) 65

M. Maggiore, Phys. Lett. B 319 (1993) 83

M. Maggiore, Phys. Rev. D 49 (1994) 5182.

[10] A. Kempf, G. Mangano and R. B. Mann, Phys. Rev. D 52 (1995) 1108.

[11] A. Kempf and G. Mangano, Phys. Rev. D 55 (1997) 7909

K. Nozari and A. Etemadi, Phys. Rev. D 85 (2012) 104029.

[12] S. K. Rama, Phys. Lett. B 519 (2001) 103

M. Lubo, Phys. Rev. D 68 (2003) 125004

K. Nozari and B. Fazlpour, Gen. Reltiv. Grav. 38 (2006) 1661

P. Wang, H. Yang and X. Zhang, JHEP 08 (2010) 043

P. Wang, H. Yang and X. Zhang, Phys. Lett. B 718 (2012) 265.

[13] L. N. Chang, D. Minic, N. Okamura and T. Takeuchi, Phys. Rev. D 65 (2002) 125028

A. F. Ali, Class. Quantum Grav. 28 (2011) 065013

P. Pedram, Phys. Lett. B 718 (2012) 638.

[14] T. Fityo, Phys. Lett. A 372 (2008) 5872.

[15] D. Mania and M. Maziashvili, Phys. Lett. B 705 (2011) 521.

[16] B. Vakili and M. A. Gorji, J. Stat. Mech. (2012) P10013.

[17] M. V. Battisti and G. Montani, Phys. Lett. B 656 (2007) 96.

[18] A. F. Ali and B. Majumder, Class. Quantum Grav. 31 (2014) 215007

[19] A. Awad and A. F. Ali, JHEP 1406 (2014) 093.

[20] S. Das and E. C. Vagenas, Phys. Rev. Lett. 101 (2008) 221301

P. Pedram, K. Nozari and S. H. Taheri, JHEP 1103 (2011) 093

S. Jalalzadeh, M. A. Gorji, K. Nozari, Gen. Reltiv. Grav. 46 (2014) 1632.

[21] M. A. Gorji, K. Nozari and B. Vakili, Phys. Rev. D 89 (2014) 084072. 